Original

\title{
Influencia del empleo de estatinas en el cáncer de próstata clinicamente localizado tratado con prostatectomia radical
}

\author{
Enrique Rijo Mora, José A. Lorente Garin, Oscar Bielsa Galí, Albert Frances Comalat, \\ Lluis Fumadó Ciutat, Octavio Arango Toro
}

Servicio de Urología. Hospital del Mar. Barcelona, España

\begin{abstract}
Resumen
Introducción: $\mathrm{El}$ empleo de estatinas se ha relacionado con la reducción del riesgo, progresión y mortalidad de algunos tumores, incluyendo el cáncer de próstata (CP). Según estudios recientes el tratamiento con estatinas a largo plazo no reduce significativamente el riesgo de padecer CP pero parece conferir un efecto protector frente al CP avanzado o agresivo. No existen datos concluyentes del efecto potencial en los pacientes diagnosticados de CP clínicamente localizado y sometidos a tratamiento radical.

Material y métodos: Se realiza un análisis retrospectivo en una cohorte de 250 pacientes no sometidos a cribado y diagnosticados en la práctica clínica de CP localizado (T1c-T2cNxM0) tratados con prostatectomía radical desde el año 2001 a 2008. Se recogen las variables clínicas y patológicas además de la existencia de dislipemia y el tratamiento con estatinas durante al menos 5 años.

Se empleó un análisis de regresión logística para determinar las variables independientes predictoras de una patología más favorable.

Resultados: En el análisis univariante hemos encontrado relación entre el tratamiento con estatinas, la presencia de carcinoma insignificante y el grupo de riesgo de recidiva bajo o intermedio. Sin embargo, está relación no es independiente en el análisis multivariante.

Discusión: La hipótesis sobre el potencial efecto de las estatinas en la prevención o la inhibición del desarrollo del cáncer de alto riesgo necesita de ensayos clínicos diseñados para analizar este objetivo.

Existen datos no concluyentes que parecen favorecer a los pacientes tratados con estatinas presentando un menor riesgo de CP de alto grado y probablemente letal.
\end{abstract}

Palabras clave: Cáncer de próstata. Estatinas. Prevención. Prostatectomía.

The impact of statins use in clinically localized prostate cancer treated with radical prostatectomy Abstract

Introduction: Use of statins has been associated with reduced risk, progression and mortality of some tumors, including prostate cancer $(\mathrm{CaP})$.

According to recent Studies, long-term treatment with statins significantly reduces the risk of PC and also confers a protective effect against advanced or aggressive CaP. However, there is no conclusive data regarding the potential effect on patients diagnosed with clinically localized CaP undergoing radical treatment.

Materials and methods: A retrospective study was performed in a cohort of 250 patients that did not undergo screening and diagnosis in clinical practice of localized CaP (T1c-T2cNxM0). All patients were treated with radical prostatectomy between 2001 and 2008. Data about clinical and pathological findings, and also the existence of dyslipemia as well as treatment with statins for at least 5 years was collected and analyzed.

A logistic regression analysis was performed to determine the independent predictive variables related with a more favorable pathology.

Results: In our study, we found a relationship, using a univariate analysis, between treatment with statins and the presence of insignificant carcinoma in the group with low or intermediate risk of recurrence. However, this relationship is not independent when we applied a multivariate analysis.

Discussion: Hypothesis about the potential effect of the statins preventing or inhibiting the development of high risk CaP needs to be proven applying specific clinical trials designed for this purpose.

There isn't conclusive data that demonstrate that statins lower the risk of development of potentially lethal high grade CaP.

Keywords: Prostate cancer. Statins. Prevention. Prostatectomy. 
$\mathrm{N}$ umerosos ensayos clínicos han demostrado un impacto significativo en la reducción de la morbimortalidad cardiovascular en pacientes dislipémicos con los inhibidores de la 3-hidroxi-3-metilglutaril coenzima A reductasa (HMG-CoA reductasa). Se ha propuesto la potencial utilidad de estos agentes en otras situaciones como el Alzheimer, la disfunción eréctil, la esclerosis múltiple o la artritis reumatoide. El empleo de estos agentes también se ha relacionado con la reducción del riesgo, progresión y mortalidad de algunos tumores, incluyendo el cáncer de próstata (CP). Según un reciente meta-análisis en el que se analizan 6 ensayos clínicos randomizados y 13 estudios observacionales, el tratamiento a largo plazo con los inhibidores de la HMG-CoA reductasa (estatinas) no reduce significativamente el riesgo de padecer $\mathrm{CP}$ pero parece conferir un efecto protector frente al CP avanzado o agresivo. Sin embargo, no existen datos epidemiológicos concluyentes del efecto potencial en los pacientes diagnosticados de CP clínicamente localizado y sometidos a tratamiento radical. Nuestro estudio evalúa el impacto del tratamiento con estatinas a largo plazo en la presentación clínica y patológica de una cohorte de pacientes diagnosticados de $\mathrm{CP}$ clínicamente localizado.

\section{MATERIAL Y MÉTODOS}

Se realiza un análisis retrospectivo en una cohorte de 250 pacientes no sometidos a cribado y diagnosticados en la práctica clínica de CP localizado (T1c-T2cNxM0) tratados con prostatectomía radical en el Hospital del Mar desde el año 2001 a 2008. Se recogen las variables clínicas y patológicas además de la existencia de dislipemia y el tratamiento con estatinas durante al menos 5 años. Las variables continuas clínicas y patológicas fueron comparadas por grupos mediante un test t de student. Las variables categóricas se compararon mediante un análisis de Chi-cuadrado. Se empleó un análisis de regresión logística (forward stepwise) para determinar las variables independientes predictoras de una patología más favorable (carcinoma clínicamente insignificante y grupos de riesgo de progresión bajo e intermedio de la NCCN). El estudio estadístico se realizó mediante el paquete estadístico SPSS versión 13.0 (SPSS, Chicago, Ill.).

\section{RESULTADOS}

Cien pacientes (40\%) presentan dislipemia definida por cifras de colesterol sérico superior a 200 $\mathrm{mg} / \mathrm{dL}$, de los cuales treinta son tratados con dieta y setenta con diferentes estatinas. No existieron diferencias estadísticamente significativas entre los pacientes que tomaban estatinas y el resto en la edad, índice de masa corporal, ingesta de AAS, PSA al diagnóstico, volumen prostático, volumen tumoral y porcentaje de tumor en la pieza quirúrgica, grado de Gleason, estadio clínico y patológico. Sí se encontraron diferencias en el análisis univariante en la clasificación por riesgos de recurrencia y en la presencia de carcinoma insignificante a favor de los consumidores de estatinas (Tablas la y 1b). Sin embargo, en el análisis de regresión logística no se demostró que el consumo de estatinas en estos pacientes fuera una variable predictora independiente de una patología más favorable (Tabla 2).

\section{DISCUSIÓN}

Swyer et al. comunicaron por primera vez que el tejido prostático tiene un alto contenido de colesterol. Schaffner et al. proporcionan la primera evidencia que reduciendo las concentraciones de colesterol se altera el crecimiento prostático. Con posterioridad, se demostró que el incremento en las concentraciones de colesterol se relacionaba con la presencia de cáncer ${ }^{1}$, identificando algunas irregularidades en la homeostasis de los lípidos como base de la acumulación de colesterol en las células prostáticas tumorales ${ }^{2}$. La próstata, en condiciones normales, sintetiza grandes cantidades de colesterol, incluso en comparación con el hígado, probablemente por tratarse de un órgano bajo control de los andrógenos. Estos han demostrado favorecer directamente la lipogénesis en las células de CP humano por activación de la transcripción de determinados genes, como la sintetasa de ácidos grasos (FAS) y la HMGCoA reductasa entre otros (Tabla 3 ).

Las estatinas, inhibidores específicos de la HMGCoA reductasa, son los agentes reductores del colesterol más prescritos en la salud cardiovascular. El mecanismo bioactivo por el que actúan es por la inhibición de la conversión del HMC-CoA a mevalonato en un paso precoz en la síntesis de colesterol, (Fig. 1). A nivel anatómico, las estatinas inhiben de forma primaria la HMG-CoA reductasa especialmente en el hígado pero apenas en la próstata. En el tumor, la vascularización aberrante contribuye a reducir la penetración tisular de las estatinas. Los efectos antitumorales no parecen ocurrir por inhibición de la síntesis de isoprenoide intratumoral, un mecanismo asumido ampliamente como base de los 
Tabla 1a. Características clínicas y patológicas de los pacientes analizados

\begin{tabular}{|c|c|c|c|c|c|c|c|}
\hline & \multicolumn{2}{|c|}{$\begin{array}{c}\text { No estatinas } \\
(\mathbf{n}=\mathbf{1 8 0})\end{array}$} & \multicolumn{2}{|c|}{$\begin{array}{c}\text { Estatinas } \\
(\mathbf{n}=70)\end{array}$} & \multirow[t]{2}{*}{$\begin{array}{c}\text { Valor de } \\
\mathbf{p}\end{array}$} & \multicolumn{2}{|c|}{$\begin{array}{c}\text { Total } \\
(\mathbf{n}=\mathbf{2 5 0})\end{array}$} \\
\hline & Mediana & $\begin{array}{l}95 \% \text { IC } \\
\text { media }\end{array}$ & Mediana & $\begin{array}{l}95 \% \text { IC } \\
\text { media }\end{array}$ & & Mediana & $\begin{array}{l}95 \% \text { IC } \\
\text { media }\end{array}$ \\
\hline \multicolumn{8}{|c|}{ Variables continuas } \\
\hline Edad (años) & 65,0 & $62,2-65,9$ & 67,0 & $63,3-67,9$ & 0,151 & 65,0 & $63,7-66,0$ \\
\hline IMC (kg/m²) & 27,5 & $26,6-27,8$ & 27,3 & $26,0-28,4$ & 0,886 & 27,4 & $26,6-27,7$ \\
\hline PSA (ng/mL) & 6,9 & $6,6-8,7$ & 6,2 & $6,1-8,0$ & 0,364 & 6,7 & $6,7-8,3$ \\
\hline $\mathrm{VP}(\mathrm{cc})$ & 49,5 & $46,9-6,7$ & 45,5 & $40,3-53,7$ & 0,198 & 48,0 & $46,4-54,3$ \\
\hline VT (cc) & 1,5 & $2,2-5,3$ & 1,6 & $1,4-3,8$ & 0,556 & 1,5 & $2,3-4,6$ \\
\hline$\%$ tumor & 2,8 & $4,8-11,2$ & 3,3 & $3,3-9,8$ & 0,849 & 3,1 & $5,2-10,0$ \\
\hline
\end{tabular}

Tabla 1b. Características clínicas y patológicas de los pacientes analizados

\begin{tabular}{|c|c|c|c|c|}
\hline & $\begin{array}{l}\text { No estatinas } \\
(\mathbf{n}=180)\end{array}$ & $\begin{array}{c}\text { Estatinas } \\
(\mathbf{n}=70)\end{array}$ & $\begin{array}{c}\text { Valor de } \\
\mathbf{p}\end{array}$ & $\begin{array}{c}\text { Total } \\
(n=250)\end{array}$ \\
\hline & n (\%) & n (\%) & & $\mathbf{n}$ \\
\hline \multicolumn{5}{|c|}{ Variables categóricas } \\
\hline \multicolumn{5}{|c|}{ Tumor palpable } \\
\hline - No & $153(85,0)$ & $55(78,6)$ & 0,222 & 208 \\
\hline$-\mathrm{Si}$ & $27(15,0)$ & $15(21,4)$ & & 42 \\
\hline \multicolumn{5}{|c|}{ Estadio patológico } \\
\hline$-\mathrm{T} 2$ & $136(75,5)$ & $46(65,6)$ & 0,116 & 182 \\
\hline - T3 & $44(24,5)$ & $24(34,4)$ & & 68 \\
\hline \multicolumn{5}{|c|}{ Grado de Gleason } \\
\hline$-<7$ & 50 & 20 & 0,757 & 70 \\
\hline-7 & 98 & 35 & & 133 \\
\hline$->7$ & 32 & 15 & & 47 \\
\hline \multicolumn{5}{|c|}{ Riesgo recurrencia } \\
\hline - Bajo & $40(22,2)$ & $28(40,0)$ & 0,009 & 68 \\
\hline - Intermedio & $124(68,8)$ & $40(57,1)$ & & 164 \\
\hline - Alto & $16(9,0)$ & $2(2,9)$ & & 18 \\
\hline \multicolumn{5}{|c|}{$\mathrm{CP}$ insignificante } \\
\hline - No & $155(86,1)$ & $52(74,3)$ & 0,026 & 207 \\
\hline - Sí & $25(13,9)$ & $18(25,7)$ & & 43 \\
\hline \multicolumn{5}{|l|}{ Masa corporal } \\
\hline - Normopeso & $38(21,1)$ & $15(21,4)$ & 0,680 & 53 \\
\hline - Sobrepeso & $102(56,6)$ & $36(51,4)$ & & 138 \\
\hline - Obesidad & $40(22,3)$ & $19(27,2)$ & & 59 \\
\hline \multicolumn{5}{|l|}{ AAS } \\
\hline - No & $162(90,0)$ & $62(88,6)$ & 0,739 & 224 \\
\hline - Sí & $18(10,0)$ & $8(11,4)$ & & 26 \\
\hline
\end{tabular}

Tabla 2. Análisis de regresión logística que analiza el consumo de estatinas como variable predictiva independiente de una patología más favorable

\begin{tabular}{lcccccc}
\hline & Beta & SE & Wald & p & Exp (B) & 95\%IC Exp (B) \\
\hline GS & -1.46 & 0.614 & 5.669 & 0.017 & 0.232 & $0.07-0.77$ \\
PSA & -1.77 & 0.887 & 6.236 & 0.03 & 0.334 & $0.16-0.87$ \\
Estatinas & 12.263 & 992.54 & 0.001 & 0.97 & 0.001 & $0-8 \mathrm{E}+206$ \\
\hline
\end{tabular}

(C. Insignificante o probabilidad baja-intermedia de recidiva) 
Tabla 3. Ejemplos de genes reguladores de los lípidos y colesterol regulados por andrógenos.

\section{Genes regulados por andrógenos}

Sintetasa de ácidos grasos (FAS)

Acetil-CoA carboxilasa

ATP citrato liasa

Estearil-CoA desaturasa

Enzima de elongación de ácidos grasos poliinsaturados de cadena larga

Ligasa de ácidos grasos de cadena larga

Hidroxilamida de ácidos grasos

Fosfatasa tipo 2a de ácido fosfatídico

Proteina transportadora de Acil-CoA

HMG-CoA sintetasa

HMG-CoA reductasa

Farnesil difosfato sintetasa

3beta-hidroxiesterol 24 reductasa

Receptor LDL

Proteina activadora de la proteina transportadora de esteroles (SREBP)

SREBP-1c

SREBP-2

\section{Función primaria}

Síntesis de ácidos grasos

Síntesis de ácidos grasos

Síntesis de ácidos grasos

Desaturación de ácidos grasos

Elongación de ácidos grasos

Activación de ácidos grasos

Señalización de lípidos

Señalización de lípidos

Transporte de ácidos grasos

Sintesis de colesterol

Síntesis de colesterol

Síntesis de colesterol

Síntesis de colesterol

Captura colesterol

Activación lipogénesis

Factor transcripción

Factor transcripción

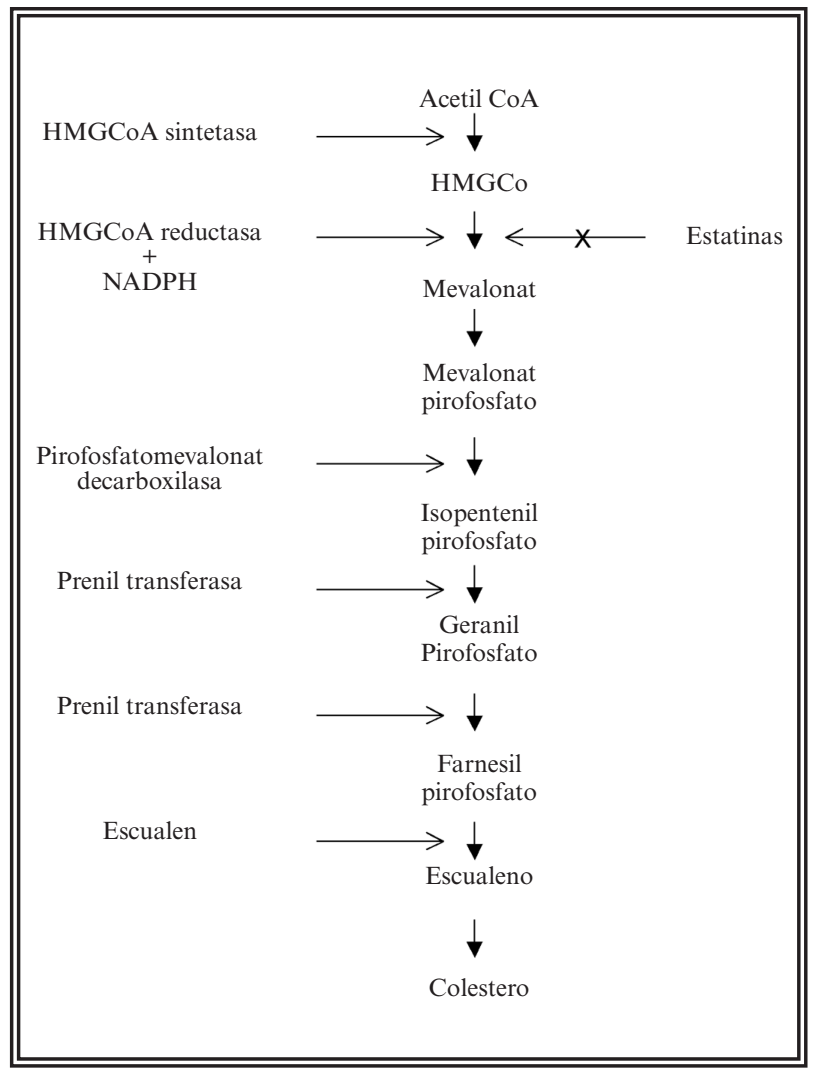

FIGURA 1. Vía de la sintesis de colesterol. El diagrama incluye las enzimas más relevantes, los mediadores de la sintesis y el punto en el que las estatinas interfieren el metabolismo de la HMGCoA. efectos de las estatinas en el cáncer. No obstante, las estatinas de uso clínico han demostrado inhibir las células tumorales y provocar apoptosis ${ }^{3-4}$. Se ha sugerido que niveles decrecientes de los mediadores de la síntesis de colesterol pueden tener efectos anti-inflamatorios, antiproliferativos e inmunomoduladores. Sin embargo, a nivel clínico, el posible efecto antitumoral está relacionado de forma indirecta con la disminución de los niveles séricos de LDL, siendo otros efectos poco probables en humanos a dosis convencionales. Por todo esto, el objetivo principal del tratamiento debe ser la reducción del colesterol sérico, ya que los individuos con valores bajos se han asociado con un menor riesgo de $\mathrm{CP}$ de alto grado y probablemente avanzado ${ }^{3}$.

No obstante, al menos dos recientes meta-análisis ponen en duda la relación positiva de esta asociación inicialmente detectada en algunos estudios de casos y controles ${ }^{5-8}$. Estos estudios concluyen que aparentemente el consumo de estatinas a bajas dosis para tratar la hipercolesterolemia no reduce el riesgo global de padecer CP. Sin embargo, no pueden descartar una reducción en el riesgo de tumor avanzado. Se han propuesto diversas aplicaciones de las estatinas en los pacientes con CP. Hipotéticamente pueden favorecer a los individuos dislipémicos afectos de CP ya que los accidentes cardiovasculares constituyen 
la primera causa de mortalidad en los pacientes con $\mathrm{CP}^{9}$. Aunque se encuentran en curso estudios dirigidos a analizar la utilidad de las estatinas en la quimioprevención, apenas existen estudios que evalúen el efecto de estos compuestos en el CP clínicamente localizado tratado con intención curativa. No hemos encontrado ningún estudio en la literatura que evalúe este aspecto en los pacientes sometidos a prostatectomía radical. Moyad et al. estudian 512 pacientes T1c-T3aNxM0 tratados con braquiterapia radical y encuentran que los pacientes tratados con estatinas presentan menor PSA inicial, porcentaje de biopsias positivas, densidad de PSA y estadio clínico. Sin embargo, esta favorable presentación no condiciona un mejor control bioquímico a 8 años de seguimiento ${ }^{10}$. En nuestro estudio, en el análisis univariante hemos encontrado relación entre el tratamiento con estatinas y la presencia de carcinoma insignificante y el grupo de riesgo de recidiva bajo o intermedio. Sin embargo, esta relación no es independiente en el análisis multivariante. Sin duda, la hipótesis sobre el potencial efecto de las estatinas en la prevención o la inhibición del desarrollo del cáncer de alto riesgo necesita de ensayos clínicos diseñados para analizar este objetivo. Analizando la literatura se desconoce el efecto en el cáncer diagnosticado en fases precoces.

En conclusión, la concienciación sobre la salud cardiovascular medida por las cifras de colesterol puede ser fácilmente olvidada, especialmente cuando se evalúan otras situaciones no directamente relacionadas. Existen, no obstante, suficientes razones para tener presente esta condición y el tratamiento con estatinas: a) la enfermedad cardiovascular es la principal causa de muerte en el mundo occidental tanto en la población general como en los participantes en los ensayos de quimioprevención para el cáncer, b) la enfermedad cardiovascular es una de las principales causas de muerte en los pacientes con $\mathrm{CP}$, c) puede existir asociación en alguno de los mecanismos que contribuyen a estos padecimientos y el CP, d) la monitorización del colesterol puede ser útil para valorar el cambio del estilo de vida en los pacientes con $\mathrm{CP}$, y e) existen datos no concluyentes que parecen favorecer a los pacientes tratados con estatinas presentando un menor riesgo de CP de alto grado y probablemente letal.

\section{REFERENCIAS}

1. Schaffner CP. Prostatic cholesterol metabolism regulation and alteration. Prog Clin Biol Res 1981;75A:279-324.

2. Swinnen JV, Van Veldhoven PP, Esquenet M, Heyns W, Verhoeven G. Androgens markedly stimulate the accumulation of neutral lipids in the human prostatic adenocarcinoma cell line LNCaP. Endocrinology 1996;137(10):4468-4474.

3. Solomon KR, Freeman MR. Do the cholesterol-lowering properties of statins affect cancer risk?. Trends Endocrinol Metab 2008;19(4):113-121.

4. Colli JL, Amling CL. High cholesterol levels are associated with reduced prostate cancer mortality rates during periods of high but not low statin use in the United States. Urol Oncol 2008; Article in Press.

5. Bonovas S, Filioussi K, Tsavaris N, Sitaras NM. Statins and Cancer Risk: A Literature-Based Meta-Analysis and MetaRegression Analysis of 35 Randomized Controlled Trials. J Clin Oncol 2006;24(30):4808-4817.

6. Bonovas S, Filioussi K, Sitaras NM. Statin use and the risk of prostate cancer: A metaanalysis of 6 randomized clinical trials and 13 observational Studies. Int J Cancer 2008;123(4):899904.

7. Agalliu I, Salinas CA, Hansten PD, Ostrander EA, Stanford JL. Statin use and risk of prostate cancer: results from a population-based epidemiologic study. Am J Epidemiol 2008;168(3): 250-260.

8. Browning DR, Martin RM. Statins and risk of cancer: A systematic review and metaanalysis. Int J Cancer 2007;120(4):833843.

9. Moyad MA, Merrick GS. Statins and cholesterol lowering after a cancer diagnosis: Why not?. Urol Oncol 2005;23(1):49-55.

10. Moyad MA, Merrick GS, Butler WM, Wallner KE, Galbreath RW, Kurko B, et al. Statins, especially atorvastatin, may favorably influence clinical presentation and biochemical progressionfree survival after Brachytherapy for clinically localized prostate cancer. Urology 2005;66(6):1150-1154.

Correspondencia autor: Dr. Enrique A. Rijo Mora Servicio de Urología. Hospital del Mar

Passeig Maritim 25-29, 08003 Barcelona. Tel.: 932483231

E-mail autor: erijo@imas.imim.es / rijo_enrique@yahoo.es

Información artículo: Original - Cáncer de próstata

Trabajo recibido: noviembre 2008

Trabajo aceptado: diciembre 2008 